Anwendungsbeispiel: Adipindialdehyd und Adipinaldehydsäure aus Cyclohexen

Ein großes U-Rohr mit Schliffhähnen an beiden Schenkeln enthält $250 \mathrm{~g}$ Kieselgel (Sorte Em [ 3 bis $5 \mathrm{~mm}$ ] der Firma Gebr. Herrmann, Köln-Ehrenfeld). Es wird an einer Ölpumpe bei $130^{\circ}$ ausgeheizt und unter Vakuum erkalten gelassen. Dann wird es in ein Kühlbad aus Kohlensäureeis/Aceton gebracht und am Ozonisator solange mit 2,5-proz. Ozon-Sauerstoffgemisch durchströmt, bis alles Gel die dunkelblaue Farbe angenommen hat. Nun werden die Hähne geschlossen und das beladene Gefäß vom Ozonisator entfernt, aber im Kühlbad belassen. Es wird mit einer Stickstoflibombe (nachgereinigter Stickstoff) verbunden und ein langsamer Stickstoffstrom hindurchgeleitet, bis der Sauerstoff aus dem Totvolumen entfernt ist. Dann verbindet man den zweiten Schenkel mit einer ebenfalls auf $-78^{\circ}$ gekühlten Glasfritten-Waschflasche, die 12,0 g Cyclohexen in $100 \mathrm{~cm}^{3}$ Methanol enthält. Man reguliert einen langsamen Gasstrom ein und hebt das U-Rohr so langsam aus dem Kühlbad heraus, daß der Gasstrom nicht zu heftig wird. Nach 4 Stdn. färbte sich die Lösung in der Vorlage leicht blau und in einer nachgeschalteten Waschflasche mit KaliumjodidLösung trat die erste Gelbfärbung auf. Das U-Rohr wird entfernt, der Ozonüberschuß mit einem kräftigen Stickstoffstrom abgeblasen und die farblose Lösung sofort in die Hydrierbirne mit vorhydriertem Palladium-Kohle-Katalysator umgefüllt. Wegen der starken Wärmeentwicklung bei der Hydrierung wurde eine Hydrierapparatur mit Wasserkühlung verwendet. Wasserstoffverbrauch $68 \%$ d. Theorie. Die vom Katalysator abfiltrierte Lösung wird eingedampft und der Rückstand fraktioniert. Man erhält $11,1 \mathrm{~g} \quad\left(66 \%\right.$ d. Th.) Adipindialdehyd, $\mathrm{Sdp}_{0,1} 55$ bis $59^{\circ}$, und $4,6 \mathrm{~g}(24 \%$ d. Th. $)$ Adipinaldehydsäure, $\operatorname{Sdp}_{0,1} 115-119^{\circ}$.

Aus 12,0 g Cycloocten wurden in entsprechender Weise $9,8 \mathrm{~g}(63 \%$ d. Th. $)$ Korkdialdehyd, $\operatorname{Sdp}_{0,1}$ $74-78^{\circ}$, und $3,5 \mathrm{~g}(20 \%$ d. Th. $)$ Korkaldehydsäure, $\operatorname{Sdp}_{0,1} 130-134^{\circ}$ erhalten.

\title{
Uber die Existenz des Perhydroxylradikals in wäßrigen Lösungen und seine Bedeutung bei Oxydationsreaktionen*
}

\author{
Von HJ. Staudinger und V. Ullirich ** \\ Aus dem Physiologisch-chemischen Institut der Justus-Liebig-Universität Gießen
}

(Z. Naturforschg. 19 b, 877-882 [1964]; eingegangen am 5. Juni 1964)

Der früher mitgeteilte Befund, daß bei der schrittweisen Reduktion von molekularem Sauerstoff durch Einelektronen-Donatoren - meist Schwermetallionen geeigneten Redoxpotentials $-\mathrm{O}_{2} \mathrm{H}$ Radikale auftreten, wird durch neue Ergebnisse weiter gestützt. Die Oxydationen über $\mathrm{O}_{2} \mathrm{H}$-Radikale verlaufen nur in Gegenwart von Reduktionsmitteln und lassen sich am besten durch den Angriff eines Sauerstoffatoms im Übergangszustand der Reaktion erklären. Die biologische Bedeutung des Mechanismus wird diskutiert.

Bei der stufenweisen Reduktion von molekularem Sauerstoff treten $\mathrm{O}_{2} \mathrm{H}$-Radikale und $\mathrm{OH}$-Radikale als reaktive $\mathrm{Zwischenprodukte} \mathrm{auf:}$

$$
\begin{aligned}
& \mathrm{O}_{2}+\mathrm{e} \rightleftharpoons \mathrm{O}_{2}^{\ominus}+\mathrm{e} \rightleftharpoons \mathrm{O}_{2}^{2} \ominus+\mathrm{e} \rightleftharpoons\left[\mathrm{O} \ominus+\mathrm{O}^{2} \ominus\right]+\mathrm{e} \rightleftharpoons 2 \mathrm{O}^{2} \ominus
\end{aligned}
$$

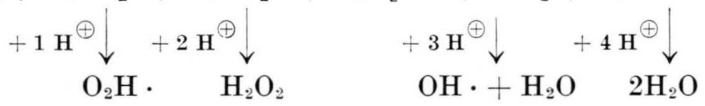

* Auszugsweise vorgetragen beim „Internationalen Symposium über Oxydasen und verwandte Enzyme" (ISOX) in Amherst (Mass., USA), July 1964.

* Aus der Dissertation von V. Ullrich, Gießen 1964.

1 F. Haber u. J. Weiss, Proc. Roy. Soc. 147, 332 [1934].

2 W. A. Waters, „The Chemistry of Free Radicals“, Oxford 1946, S. 247.

3 C. N. Hinshelwood, Proc. Roy. Soc. 188, 1 [1946].

4 R. G. W. Norrish, Z. Elektrochem. 56, 705 [1952].
Hydroxylradikale entstehen bei der Reduktion des Wasserstoffperoxyds ${ }^{1,2}$. Sie können auch bei Reaktionen von Sauerstoff mit atomarem Wasserstoff ${ }^{3-5}$ sowie bei der Einwirkung energiereicher Strahlung auf $\mathrm{H}_{2} \mathrm{O}_{2}{ }^{6,7}$ oder $\mathrm{H}_{2} \mathrm{O}^{8,9}$ nachgewiesen werden. Ihre relativ lange Lebensdauer gestattete die Untersuchung der physikalischen ${ }^{4}$ und chemischen ${ }^{10}$ Eigenschaften.

5 S. N. Foner u. R. L. Hudson, J. chem. Physics 23, 1974 [1955].

${ }^{6}$ H. C. Urey, L. H. Dawsey u. F. O. Rice, J. Amer. chem. Soc. 1371 [1929].

7 G. Stein u. J. Weiss, Nature [London] 166, 1104 [1950].

8 H. Loebl, G. Stein u. J. Weiss, J. chem. Soc. [London] 1950, 2704.

${ }^{9}$ G. Stein u. J. Weiss, J. chem. Soc. [London] 1951, 3255.

10 G. G. Jayson, G. Scholes u. J. Weiss, J. chem. Soc. [London] 1957, 1358 (dort weitere Literaturangaben). 
Die Existenz des Perhydroxylradikals wurde zuerst bei Reaktionen in der Gasphase vermutet und später auch nachgewiesen ${ }^{4,5}$; eine Beteiligung bei chemischen Reaktionen in Lösung konnte bisher in einer Reihe von Fällen nur hypothetisch angenommen werden ${ }^{11-22}$.

OH-Radikale sind als hydroxylierende Teilchen eingehend untersucht ${ }^{10,18,23}$. Sie entstehen in guter Ausbeute bei der Reduktion von $\mathrm{H}_{2} \mathrm{O}_{2}$ mit dem $\mathrm{Fe}^{2 \oplus}$ EDTA *-Komplex und bei ihrem Angriff auf Acetanilid entstehen die drei möglichen $N$-Acetylaminophenole im Verhältnis $o=46,5 \%, m=2,5 \%$ und $p=51$ Prozent $^{24}$. Diese Verteilung der Hydroxylierungsprodukte läßt auf einen elektrophilen Charakter der Reaktion schließen.

Reduziert man molekularen Sauerstoff mit dem $\mathrm{Fe}^{2 \oplus}$ EDTA-Komplex in Gegenwart von Acetanilid, so beobachtet man ebenfalls die Entstehung von $N$. Acetylaminophenolen, aber im Isomerenverhältnis 33\% : 14\%: 53 Prozent. Wird zur Reduktion des Sauerstoffs Titan(III) -chlorid eingesetzt, verschiebt sich das Verhältnis zu den Werten 35\%:33\%: 32 Prozent ${ }^{24}$.

Diese von den Ergebnissen der Hydroxylierung mit Fentons Reagens starken Abweichungen machten wahrscheinlich, daß unter bestimmten Voraussetzungen an der Hydroxylierung mit molekula: rem Sauerstoff ein anderes Teilchen als das $\mathrm{OH}$ Radikal, also vermutlich das $\mathrm{O}_{2} \mathrm{H}$-Radikal, beteiligt ist.

Die Ergebnisse der vorliegenden Arbeit bestätigen diese Annahme und lassen es als sicher erscheinen, daß die Hydroxylierung über $\mathrm{O}_{2} \mathrm{H}$-Radikale für das Auftreten eines $o: m: p$-Verhältnisses von etwa $40: 40: 20$ beim Acetanilid verantwortlich ist.

11 G. J. Minkoff, Discuss. Faraday Soc. 2, 151 [1947] (dort weitere Literaturangaben).

12 H. Loebl, G. Stein u. J. Weiss, J. chem. Soc. [London] 1949, 2074.

13 G. Stein u. J. Weiss, J. chem. Soc. [London] 1949, 3245.

14 G. R. A. Johnson, G. Scholes u. J. Weiss, J. chem. Soc. [London] 1953, 3091.

15 G. G. Jayson, G. Scholes u. J. Weiss, J. Biochemistry 57, 386 [1954].

16 I. M. Kolthoff u. J. Jordan, Analytic. Chem. 24, 1071 [1952].

17 I. Fredovich u. P. Handler, J. biol. Chemistry 236, 1836 [1961].

18 R. O. C. Norman u. G. K. Radda, Proc. chem. Soc. [London] 1962, 138.

19 A. Rieche, Angew. Chem. 75, 870 [1963].

\section{Methoden}

Die Bestimmung der $o$-, $m$ - und $p$-Hydroxylierungsprodukte des Acetanilids ist von uns ausführlich beschrieben worden ${ }^{24,25}$. Die Versuchsbedingungen der Ansätze sind den Legenden der Tabellen zu entnehmen.

\section{Ergebnisse}

\section{a) Nachweis einer radikalischen Hydroxylierung} im System $T i^{3 \oplus} / \mathrm{O}_{2}$

Die Hydroxylierung des Acetanilids wird im $\mathrm{Ti}^{3 \oplus} / \mathrm{O}_{2}$-System durch Sulfit, das als Fänger für Sauerstoffradikale beschrieben worden ist ${ }^{17,26}$, und durch Hydrochinon fast vollständig gehemmt. Ebenso muß die Katalyse der Polymerisation von Methacrylsäuremethylester ${ }^{27,28}$ als eindeutiger Hinweis für das Auftreten von freien Radikalen in diesem System angesehen werden.

Ein direkter Nachweis des Perhydroxylradikals durch Abfangreaktionen und Isolierung der entstehenden Peroxyde ist in Gegenwart der starken Reduktionsmittel nicht möglich. Es wurde auf anderem Wege versucht seine Beteiligung an der Hydroxylierung zu beweisen.

\section{b) Abfangreaktionen für $\mathrm{H}_{2} \mathrm{O}_{2}$}

Nach unserer Vermutung wird im $\mathrm{Ti}^{3 \oplus} / \mathrm{O}_{2}$-System das intermediär entstehende $\mathrm{H}_{2} \mathrm{O}_{2}$ als Titan-Peroxyd-Komplex abgefangen ${ }^{24}$. Eine Hydroxylierung durch $\mathrm{OH}$-Radikale, die nur über eine Reduktion des $\mathrm{H}_{2} \mathrm{O}_{2}$ auftreten können, ist damit blockiert. Der charakteristisch hohe Anteil an $N$-Acetyl- $m$-aminophenol muß in diesem Fall durch einen Angriff über das Perhydroxylradikal erklärt werden.

20 I. Yamazaki u. L. H. Piette, Biochim. biophysica Acta [Amsterdam] 77, 47 [1963].

21 A. L. Tappel, P. D. Boyer u. W. O. Lundberg, J. biol. Chemistry 199, 267 [1952].

22 H. S. Mason, Science [Washington] 125, 1185 [1957].

* EDTA = Dinatriumsalz der Äthylendiamintetraessigsäure.

23 R. Breslow u. L. N. Lukens, J. biol. Chemistry 235, 292 [1960].

24 H.. Staudinger u. V. Ullrich, Z. Naturforschg. 19 b, 409 [1964].

25 HJ. Staudinger u. V. Ullrich, Biochem. Z. 339, 491 [1964].

26 I. Fredovich u. P. Handler, J. biol. Chemistry 233, 1578 [1958].

27 R. M. Acheson u. C. M. Hazelwood, Biochim. biophysica Acta [Amsterdam] 42, 49 [1960].

28 V. Ullrich, Dissertation Gießen 1964. 


\begin{tabular}{|c|c|c|c|c|c|}
\hline \multirow[b]{2}{*}{ System } & \multirow[b]{2}{*}{ Zusatz } & \multicolumn{4}{|c|}{$N$-Acetylaminophenol } \\
\hline & & $\begin{array}{c}o- \\
{[\%]}\end{array}$ & $\begin{array}{c}m- \\
{[\%]}\end{array}$ & $\begin{array}{c}p- \\
{[\%]}\end{array}$ & $\begin{array}{c}\text { Ausbeuten } \\
{[\mu \mathrm{Mol}]}\end{array}$ \\
\hline $\mathrm{Ti}^{3} \oplus / \mathrm{O}_{2}^{24}$ & & 35 & 33 & 32 & 0,90 \\
\hline $\mathrm{Fe}^{3 \oplus / \mathrm{EDTA} / \mathrm{Asc} / \mathrm{O}_{2}}$ & & 35 & 10 & 55 & 2,50 \\
\hline 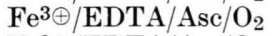 & Katalase & 37 & 32 & 31 & 1,50 \\
\hline 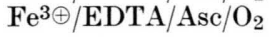 & $\mathrm{MnO}_{2}$ & 36 & 22 & 42 & 0,25 \\
\hline
\end{tabular}

Tab. 1. Abfangreaktionen für $\mathrm{H}_{2} \mathrm{O}_{2} .5 \mu \mathrm{Mol} \mathrm{Fe}{ }_{2}\left(\mathrm{SO}_{4}\right)_{3} \cdot 9 \mathrm{H}_{2} \mathrm{O}, 8 \mu \mathrm{Mol}$ EDTA, $30 \mu \mathrm{Mol}$ Ascorbinsäure, $50 \mu \mathrm{Mol}$ Acetanilid (ges. Lösung) ; mit Tris-Puffer $p_{\mathrm{H}} 7,4$ auf 1,0 ml auffüllen. Z usätze: $500 \mathrm{E}^{29}$ Katalase (Boehringer), $100 \mu \mathrm{Mol} \mathrm{MnO}_{2}$ (gefällt und getrocknet). Temperatur: $20^{\circ}$; Zeit: 60 Minuten. Die Werte sind Mittel aus drei Versuchen.

Als experimentelle Stütze für diese Annahme kann der Nachweis des Titan-Peroxyd-Komplexes angesehen werden, der beim Ansäuern einer mit Sauerstoff oxydierten Titan(III) -chlorid-Lösung an seiner gelben Farbe leicht erkannt wird.

Auf analogem Wege ist durch Abfangen von $\mathrm{H}_{2} \mathrm{O}_{2}$ im System $\mathrm{Fe}^{2 \oplus} \mathrm{EDTA} / \mathrm{O}_{2}$ eine Beeinflussung der $o: m: p$-Relationen zu erreichen. Die Versuche in Tab. 1 zeigen die Wirkung von Katalase und Braunstein auf die Hydroxylierung des Acetanilids.

Nach Zusatz von Katalase werden etwa die gleichen $o: p: m$-Relationen wie im Titan-System erhalten. Durch die Katalase wird entstandenes $\mathrm{H}_{2} \mathrm{O}_{2}$ zersetzt und auch hier das Auftreten von $\mathrm{OH}$-Radikalen verhindert. Damit findet der hohe Anteil an $N$-Acetyl- $m$-aminophenol wiederum über einen Angriff des $\mathrm{O}_{2} \mathrm{H}$-Radikals seine Erklärung. In gleichem Sinne trägt die bekannte $\mathrm{H}_{2} \mathrm{O}_{2}$-zersetzende Wirkung von Mangandioxyd zur Steigerung der $m$-Hydroxylierung bei.

\section{c) Temperaturabhängigkeit der Hydroxylierungs- Reaktionen}

Tab. 2 zeigt den Einfluß der Temperatur auf die Hydroxylierung des Acetanilids. Den Erwartungen entsprechend ist mit dem Fenton-Reagens das $o: m: p$-Verhältnis der $N$-Acetylaminophenole von der Temperatur unabhängig und bestätigt damit einen einheitlichen Hydroxylierungs-Mechanismus durch das OH-Radikal.

Eine ausgeprägte Temperaturabhängigkeit findet man dagegen bei der Hydroxylierung mit molekularem Sauerstoff (System $\mathrm{Fe}^{2 \oplus}{ }^{\oplus} \mathrm{EDTA} / \mathrm{O}_{2}$ ), die durch die Beteiligung eines zweiten Mechanismus erklärt werden kann. Bei tiefer Temperatur nähern sich die Isomerenverhältnisse der entstehenden $N$-Acetylaminophenole den Werten, die man im Titan-System erhält. Die Ursache für dieses Ergebnis kann eine Stabilisierung des $\mathrm{O}_{2} \mathrm{H}$-Radikals bei tiefer Temperatur oder eine geringere Aktivierungsenergie der $\mathrm{O}_{2} \mathrm{H}$-Hydroxylierung sein. Die Unbeständigkeit des $\mathrm{O}_{2} \mathrm{H}$-Radikals ist in seiner hohen Reaktivität begründet. Es kann durch die anwesenden starken Reduktionsmittel weiterreduziert werden [Gl. (1)] oder nach folgenden Gln. reagieren :

$$
\begin{aligned}
& 2 \mathrm{O}_{2} \mathrm{H} \cdot \rightarrow \mathrm{O}_{2}+\mathrm{H}_{2} \mathrm{O}_{2} \\
& \mathrm{H}_{2} \mathrm{O}_{2}+\mathrm{O}_{2} \mathrm{H} \cdot \rightarrow \mathrm{H}_{2} \mathrm{O}+\mathrm{O}_{2}+\mathrm{OH} \cdot \\
& \mathrm{OH} \cdot+\mathrm{O}_{2} \mathrm{H} \cdot \rightarrow \mathrm{H}_{2} \mathrm{O}+\mathrm{O}_{2} \\
& \mathrm{Me}_{\mathrm{Ox}}+\mathrm{O}_{2} \mathrm{H} \cdot \rightarrow \mathrm{Me}_{\mathrm{red}}+\mathrm{O}_{2} .
\end{aligned}
$$

Im $\quad \mathrm{Ti}^{3 \oplus} / \mathrm{O}_{2}$-System nimmt $N$-Acetyl- $m$-aminophenol nur noch geringfügig zu und bestätigt unsere

\begin{tabular}{|c|c|c|c|c|c|c|c|c|c|c|c|c|}
\hline \multirow{2}{*}{$\begin{array}{c}t \\
{\left[{ }^{\circ} \mathrm{C}\right]}\end{array}$} & \multicolumn{4}{|c|}{$\begin{array}{c}\mathrm{Fe}^{2 \oplus / \mathrm{EDTA} / \mathrm{O}_{2}} \\
N \text {-Acetylaminophenol }\end{array}$} & \multicolumn{4}{|c|}{$\begin{array}{c}\mathrm{Ti}^{3} \oplus / \mathrm{O}_{2} \\
N \text {-Acetylaminophenol }\end{array}$} & \multicolumn{4}{|c|}{$\begin{array}{c}\mathrm{Fe}^{2 \oplus} / \mathrm{EDTA} / \mathrm{H}_{2} \mathrm{O}_{2} \\
N \text {-Acetylaminophenol }\end{array}$} \\
\hline & $\begin{array}{c}o- \\
{[\%]}\end{array}$ & $\begin{array}{c}m- \\
{[\%]}\end{array}$ & $\begin{array}{c}p- \\
{[\%]}\end{array}$ & {$[\mu \mathrm{Mol}]$} & $\begin{array}{c}o- \\
{[\%]}\end{array}$ & $\begin{array}{c}m- \\
{[\%]}\end{array}$ & $\begin{array}{c}p- \\
{[\%]}\end{array}$ & {$[\mu \mathrm{Mol}]$} & $\begin{array}{c}o- \\
{[\%]}\end{array}$ & $\begin{array}{c}m- \\
{[\%]}\end{array}$ & $\begin{array}{c}p- \\
{[\%]}\end{array}$ & {$[\mu \mathrm{Mol}]$} \\
\hline 100 & 45 & 5 & 50 & 1,77 & 33 & 30 & 37 & 0,10 & 44 & 3 & 53 & 1,67 \\
\hline 50 & 40 & 9 & 51 & 1,35 & 29 & 33 & 38 & 0,60 & 43 & 3 & 54 & 1,50 \\
\hline 20 & 36 & 14 & 50 & 1,25 & 35 & 33 & 32 & 1,13 & 47 & 3 & 50 & 1,38 \\
\hline & 28 & 24 & 48 & 0,77 & 31 & 35 & 34 & 1,25 & 48 & 3 & 49 & 1,13 \\
\hline-10 & 33 & 29 & 38 & 0,49 & 35 & 36 & 29 & 0,40 & 48 & 4 & 48 & 1,03 \\
\hline
\end{tabular}
Vermutung, daß in diesem System bereits bei höherer Temperatur die Hydroxylierung über das $\mathrm{O}_{2} \mathrm{H}$ Radikal überwiegt.

Tab. 2. Temperaturabhängigkeit der Hydroxylierungen. $65 \mu \mathrm{Mol} \mathrm{FeSO}_{4} \cdot 7 \mathrm{H}_{2} \mathrm{O} ; 100 \mu \mathrm{Mol}$ EDTA, $200 \mu \mathrm{Mol}_{\mathrm{TiCl}}$-Lösung (15-proz., eisenfrei, Merck), vor Inkubation auf $p_{\mathrm{H}}$ 5-6 einstellen, $100 \mu \mathrm{Mol}$ Acetanilid/0,2 ml Acetonitril. Mit 0,1-m. Acetatpuffer $p_{\mathrm{H}} 5,8$ auf $1,0 \mathrm{ml}$ Gesamtvolumen auffüllen. 20 min $\mathrm{O}_{2}$ bzw. im System $\mathrm{Fe}^{2 \oplus} / \mathrm{EDTA} / \mathrm{H}_{2} \mathrm{O}_{2}$ superreinen $\mathrm{N}_{2}$ durchperlen. Alle Werte sind Mittel aus drei Versuchen. 


\begin{tabular}{|c|c|c|c|c|c|c|c|c|c|c|c|c|}
\hline \multirow{2}{*}{$p \mathrm{H}$} & \multicolumn{4}{|c|}{$\begin{array}{c}\mathrm{Fe}^{2 \oplus / \mathrm{EDTA} / \mathrm{O}_{2}} \\
N \text {-Acetylaminophenol }\end{array}$} & \multicolumn{4}{|c|}{$\begin{array}{c}\mathrm{Ti}^{3 \oplus} / \mathrm{O}_{2} \\
N \text {-Acetylaminophenol }\end{array}$} & \multicolumn{4}{|c|}{$\begin{array}{c}\mathrm{Fe}^{2 \oplus} / \mathrm{EDTA} / \mathrm{H}_{2} \mathrm{O}_{2} \\
N \text {-Acetylaminophenol }\end{array}$} \\
\hline & $\begin{array}{c}o- \\
{[\%]}\end{array}$ & $\begin{array}{c}m- \\
{[\%]}\end{array}$ & $\begin{array}{c}p- \\
{[\%]}\end{array}$ & {$[\mu \mathrm{Mol}]$} & $\left.\begin{array}{c}o- \\
{[\%}\end{array}\right]$ & $\begin{array}{c}m- \\
{[\%]}\end{array}$ & $\begin{array}{c}p- \\
{[\%]}\end{array}$ & {$[\mu \mathrm{Mol}]$} & $\begin{array}{c}O- \\
{[\%]}\end{array}$ & $\begin{array}{c}m- \\
{[\%]}\end{array}$ & $\begin{array}{c}p- \\
{[\%]}\end{array}$ & {$[\mu \mathrm{Mol}]$} \\
\hline 3,0 & 39 & 22 & 39 & 0,80 & 40 & 40 & 20 & 0,90 & 46 & 3 & 51 & 0,51 \\
\hline 4,5 & 37 & 18 & 45 & 1,15 & 41 & 38 & 21 & 0,63 & 46 & 4 & 50 & 0,86 \\
\hline 5,7 & 38 & 14 & 48 & 1,32 & 41 & 40 & 19 & 0,54 & 48 & 3 & 49 & 1,88 \\
\hline 7,4 & 40 & 13 & 47 & 0,90 & 42 & 37 & 21 & 0,19 & 48 & 3 & 49 & 1,05 \\
\hline 8,5 & 42 & 10 & 48 & 1,92 & 34 & 33 & 33 & 0,26 & 49 & 3 & 48 & 1,82 \\
\hline
\end{tabular}

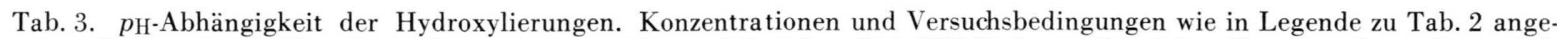
geben. Puffer: 0,1-m. Phosphatpuffer $p_{\mathrm{H}} 3,0 ; p_{\mathrm{H}} 4,5 ; p_{\mathrm{H}}$ 5,7. 0,1- $m$. Tris-HCl-Puffer $p_{\mathrm{H}}$ 7,4; 0,1-m. Pyrophosphatpuffer $p_{\mathrm{H}} 8,5$. Die Werte sind Mittel aus zwei Versuchen.

\section{d) $p_{\mathrm{H}^{-}}$Abhängigkeit der Hydroxylierungs- Reaktionen}

Hydroxylierungen im F e n t o n - System sind $p_{\mathrm{H}^{-}}$ unabhängig, da das $\mathrm{OH}$-Radikal eine extrem kleine Dissoziationskonstante besitzt. Für das Perhydroxylradikal dagegen wurde ein $p_{\mathrm{K}}$-Wert von etwa 3 berechnet ${ }^{30-32}$.

$$
\mathrm{O}_{2}{ }^{\ominus}+\mathrm{H}^{\oplus} \rightleftarrows \mathrm{O}_{2} \mathrm{H} \quad p_{\mathrm{K}} 3 \text {. }
$$

Damit ist nach Gl. (6) die Konzentration der $\mathrm{O}_{2} \mathrm{H}$ Radikale von der Protonenkonzentration abhängig. Eine $p_{\mathrm{H}}$-Änderung äußert sich in einer Verschiebung der $o: m: p$-Relationen der $N$-Acetylaminophenole im $\mathrm{Fe}^{2 \oplus}{ }^{\oplus} \mathrm{EDTA} / \mathrm{O}_{2}$-System.

Mit abnehmendem $p_{\mathrm{H}}$ nimmt die Hydroxylierung in $m$-Stellung relativ zu, während sich in den Kontrollversuchen mit dem F e n to n-System die Relationen nicht ändern. Die Gesamtausbeuten an Phenolen verringern sich im Titan-System im Gegensatz zu den Ausbeuten in den anderen Systemen mit steigendem $p_{\mathrm{H}}$; ebenfalls ein Hinweis dafür, daß die Konzentration der $\mathrm{O}_{2} \mathrm{H}$-Radikale mit steigendem $p_{\mathrm{H}}$ abnimmt.

\section{a) o:m:p:-Relationen der reinen ${ }_{,} \mathrm{O}_{2} \mathrm{H}$ - Hydroxylierung"}

Auf Grund der mitgeteilten Ergebnisse der Temperatur- und $p_{\mathrm{H}}$-Abhängigkeit kann man erwarten, daß die beste Annäherung an die reinen $o: m: p$-Relationen der $\mathrm{O}_{2} \mathrm{H}$-Hydroxylierung bei tiefer Temperatur und niedrigem $p_{\mathrm{H}}$ zu beobachten sein wird.

Sowohl mit dem $\mathrm{Ti}^{3 \oplus}{ }^{\oplus}$-System als auch mit dem

${ }^{29}$ H. U. Bergmeyer, Biochem. Z. 327, 255 [1955].

30 H. Taube, J. Amer. chem. Soc. 64, 2468 [1942].

31 J. H. Baxendale, W. G. Barb, P. George u. K. R. Hargrave, Trans. Faraday Soc. 47, 462, 591 [1951].

\begin{tabular}{|l|c|c|c|c|}
\hline \multicolumn{1}{|c|}{ System } & \multicolumn{4}{|c|}{$N$-Acetylaminophenol } \\
& $\begin{array}{c}o- \\
{[\%]}\end{array}$ & $\begin{array}{c}m- \\
{[\%]}\end{array}$ & $\begin{array}{c}p- \\
{[\%]}\end{array}$ & $\begin{array}{c}\text { Ausbeute } \\
{[\mu \mathrm{Mol}]}\end{array}$ \\
\hline $\mathrm{Ti}^{3+} / \mathrm{O}_{2}$ & 37 & 39 & 24 & 0,40 \\
$\mathrm{Fe}^{2+} / \mathrm{EDTA} / \mathrm{O}_{2}$ & 37 & 39 & 24 & 0,65 \\
\hline
\end{tabular}

Tab. 4. $o: m: p$-Relationen bei der ${ } \mathrm{O}_{2} \mathrm{H}$-Hydroxylierung “ von Acetanilid. Konzentrationen wie in Legende zu Tab. 2 angegeben. Inkubation bei $p_{\mathrm{H}} 3,0(0,1-\mathrm{m}$. Phosphatpuffer) und $-10^{\circ} \mathrm{C}$.

$\mathrm{Fe}^{2 \oplus}$ EDTA-System entstehen $o$-, $m$ - und $p$ - $N$-Acetylaminophenole in gleichen Isomerenverhältnissen. Daraus kann gefolgert werden, daß unter diesen Bedingungen nur das $\mathrm{O}_{2} \mathrm{H}$-Radikal an der Hydroxylierungs-Reaktion beteiligt ist. Die erhaltenen Werte entsprechen beinahe den theoretisch für eine Radikalreaktion zu erwartenden $(40: 40: 20)$. Im Gegensatz dazu hydroxylieren OH-Radikale aromatische Verbindungen in $o: m: p$-Verhältnissen, die für eine elektrophile Reaktion charakteristisch sind ${ }^{18}$.

\section{Diskussion}

\section{a) Mechanismus der Hydroxylierung über $\mathrm{O}_{2} \mathrm{H}$-Radikale}

Hydroxylierungen aromatischer Verbindungen mit $\mathrm{OH}$-Radikalen verlaufen über eine Addition des Radikals an die $\pi$-Elektronenwolke des Kerns ${ }^{33,34}$. Die elektrophile Orientierung der Hydroxylgruppe wird dadurch verständlich ${ }^{28,24}$.

Die hier beschriebenen Hydroxylierungen mit $\mathrm{O}_{2} \mathrm{H}$-Radikalen zeigen eine völlig andere, fast statistische Verteilung der entstehenden Phenole, die

32 J. WeISS, Trans. Faraday Soc. 31, 668 [1935].

33 J. R. Lindsay Smith u. R. O. C. Norman, J. chem. Soc. [London] 1963, 2697.

34 W. T. Dixon u. R. O. C. Norman, Proc. chem. Soc. [London] 1963, 97. 
nicht durch eine Addition an den Kern, sondern nur durch eine Substitution des Kernwasserstoffs erklärt werden kann. Eine Spaltung der $\mathrm{C}-\mathrm{H}$-Bindung durch das $\mathrm{O}_{2} \mathrm{H}$-Radikal selbst, ohne Beteiligung eines Reduktionsmittels, läßt sich experimentell nicht nachweisen ${ }^{28,35}$ und ist auch energetisch nicht möglich ${ }^{36}$. Hydroxylierungen mit $\mathrm{O}_{2} \mathrm{H}$-Radikalen verlaufen nur bei gleichzeitiger Anwesenheit eines starken Elektronendonators nach der Summengleichung:

$$
\mathrm{AH}+\mathrm{O}_{2} \mathrm{H}+\mathrm{e}=\mathrm{AOH}+\mathrm{OH}^{\ominus} .
$$

Es gibt experimentelle Hinweise dafür, daß diese Oxydationen durch atomaren Sauerstoff erfolgen, der in einem Übergangszustand der Reaktion auftritt:

So wird das Epoxyd des Cyclohexens bei der Hydroxylierung mit dem $\mathrm{Fe}^{2 \oplus}$ EDTA-Komplex, Ascorbinsäure und Sauerstoff (Ud e n f r i e n d-System) erhalten ${ }^{37}$. Bei der Inkubation von Naphthalin mit dem Eisen(II) -Pyrophosphat-Komplex und Sauerstoff fanden wir als Hauptprodukt neben $\alpha$-Naphthol das 1.2-Dihydro-diol des Naphthalins ${ }^{28}$. Seine Entstehung ist nur über die Zwischenstufe des Epoxyds und dessen Hydrolyse zu erklären. Diese Verbindung bildet sich nicht mit $\mathrm{H}_{2} \mathrm{O}_{2}$ oder mit $\mathrm{OH}$-Radikalen. Da auch $\mathrm{OH}^{\oplus}$-Ionen mit Sicherheit auszuschließen sind ${ }^{27,28,35}$, bleibt nur die Möglichkeit einer Beteiligung von Sauerstoffatomen anzunehmen.

Die Reaktion:

$$
\mathrm{O}_{2} \mathrm{H}+\mathrm{e}+\mathrm{H}^{\oplus}=\cdot \overline{\mathbf{0}} \cdot+\mathrm{H}_{2} \mathrm{O}
$$

ist aber gegenüber der Konkurrenzreaktion:

$$
\mathrm{O}_{2} \mathrm{H}+\mathrm{e}+\mathrm{H}^{\oplus}=\mathrm{H}_{2} \mathrm{O}_{2}
$$

energetisch benachteiligt ${ }^{38}$.

Man muß deshalb annehmen, daß Sauerstoffatome nur in gekoppelter Reaktion mit der Oxydation eines geeigneten Substrates angreifen können.

35 R. O. C. Norman u. J. R. Lindsay Smith, „Int. Symposium über Oxydasen u. verwandte Enzyme", Amherst (Mass., USA) 1964.

36 G. Stein u. J. Weiss, J. chem. Soc. [London] 1949, 3245.

37 G. A. Hamilton, L. Woo u. J. R. Workman, 146th Amer. chem. Soc. Meeting, Denver, Colorado, 1964, S. 13 A.

38 P. GEorge, „Int. Symposium über Oxydasen und verwandte Enzyme“, Amherst (Mass., USA) 1964.

39 M. Hayano, in: O. Hayaishi, „Oxygenases“ Academic Press, N.Y.-London 1962, S. 181.

40 J. W. Foster, in: O. Hayaishi, „Oxygenases", Academic Press, N.Y.-London 1962, S. 241.

\section{b) Biologische Bedeutungen der Oxydationen über $\mathrm{O}_{2} \mathrm{H}$-Radikale}

Bei vielen biologischen Oxydationen wird ein Atom des molekularen Sauerstoffs in das Substrat eingebaut, während das zweite zu Wasser reduziert wird. Die Enzyme, die diese Reaktionen katalysieren, werden unter dem Begriff „mischfunktionelle Oxygenasen “ zusammengefaßt. Neben den enzymatischen Hydroxylierungen gehören die enzymatischen $O-, N$ - und $S$-Desalkylierungen, die Desaminierungen, die Oxydationen von Schwefel und Stickstoff sowie die Seitenketten-Oxydationen zu diesem Reaktionstyp. Isolierte Doppelbindungen werden von diesen Enzymen bevorzugt zu Epoxyden ${ }^{30}$ und Dihydrodiol-Verbindungen ${ }^{40}$ oxydiert.

Alle erwähnten Oxydationen lassen sich auch mit molekularem Sauerstoff und dem Eisen(II)-pyrophosphat-Komplex durchführen ${ }^{28}$.

In Modellsystemen zur enzymatischen Hydroxy. lierung mit autoxydablen Cytochromen, reduziertem Pyridinnucleotid und Flavinenzymen als Kofaktoren, verläuft die Einführung der $\mathrm{OH}-\mathrm{Gruppe}$ in den aromatischen Kern zum größten Teil über $\mathrm{O}_{2} \mathrm{H}$ Radikale ${ }^{25}$.

Auch die Hydroxylierungen mit dem PeroxydaseDihydroxyfumarat-System gehorchen diesem Mechanismus ${ }^{20,41}$.

Autoxydable Eisen- und Kupferkomplexe sind die biologischen Katalysatoren für die Reduktion von Sauerstoff und können nach dem gleichen Mechanismus Perhydroxylradikale bilden.

Von dem $\mathrm{Fe}^{2 \oplus}$ - und dem $\mathrm{Cu}^{\oplus}$.Ion sind Additionsverbindungen mit Sauerstoff bekannt, die als Perferrylion bzw. Percuprylion bezeichnet werden ${ }^{39-43}$. Das folgende Formelschema veranschaulicht die Bildung und Stabilisierung der $\mathrm{O}_{2} \mathrm{H}$-Radikale:

$$
\begin{aligned}
\mathrm{Fe}^{2 \oplus}+\mathrm{O}_{2} \rightleftarrows\left[\mathrm{Fe}^{2 \oplus} \mathrm{O}_{2}\right]^{2 \oplus} \leftarrow & \rightarrow \mathrm{I} \\
{\left[\mathrm{Fe}^{3 \oplus} \mathrm{O}_{2}{ }^{\ominus}\right]^{2 \oplus} } & \rightleftharpoons \mathrm{Fe}^{3 \oplus}+\mathrm{O}_{2} \stackrel{\ominus}{\mathrm{II}} \stackrel{\mathrm{H}}{\rightarrow} \mathrm{O}_{2} \mathrm{H} .
\end{aligned}
$$

41 D. Hey, Hu. Staudinger u. V. Ullrich, in Vorbereitung.

42 L. H. Voght, H. M. Faigenbaum u. S. E. Wiberley, Chem. Reviews 63, 269 [1963].

43 G. Graaf u. S. Fallab, Experientia [Basel] XX, 46 [1964].

44 Ll. L. Ingraham, „Biochemical Mechanisms“, J. Wiley \& Sons Inc., N.Y.-London 1962, S. 68.

45 P. George u. J. S. Griffith, in: Boyer, Lardy, Myrbäck "The Enzymes"Vol. I, S. 347.

46 R. J. P. Williams, in: Boyer, Lardy, Myrbäck „The Enzymes"Vol. I. S. 391. 
Die Bindung des Sauerstoffs erfolgt in der Art eines Elektronen-Donator-Akzeptor-Komplexes (I), bei dem durch "charge transfer" das $\mathrm{O}_{2}{ }^{\ominus}$-Ion aus der Grenzstruktur II heraus entstehen kann und nach Aufnahme eines Protons in das freie Radikal übergeht.
Weitere Arbeiten sollen die biologische Bedeutung der Oxydationen über $\mathrm{O}_{2} \mathrm{H}$-Radikale stützen.

Der Deutschen Forschungsgemeinschaft danken wir für ihre wertvolle Unterstützung.

\title{
Thermische und photochemische Polymerisation von Acrylsäureäthylester mit Metallcarbonyl-Derivaten
}

\author{
Von W. Strohmeier und P. Hartmann \\ Aus dem Institut für Physikalische Chemie der Universität Würzburg \\ (Z. Naturforschg. 19 b, 882-886 [1964] ; eingegangen am 29. Juni 1964)
}

\begin{abstract}
Im Gegensatz zur thermischen Polymerisation kann reines Äthylacrylat (ÄA) photochemisch mit großer Ausbeute auch unterhalb $100^{\circ} \mathrm{C}$ zu festen, zähen Polymeren mit hohem Mol.-Gew. poly. merisiert werden.

Zusatz von Metallcarbonylen zu reinem $\mathrm{AA}$ ergab für $\mathrm{Cr}(\mathrm{CO})_{6}$ und $\mathrm{W}(\mathrm{CO})_{6}$ eine Erhöhung der Polymerisations-Geschwindigkeit, während $\mathrm{Mo}(\mathrm{CO})_{6}, \mathrm{Mo}(\mathrm{CO})_{5} \mathrm{Pyridin}_{6} \mathrm{Fe}(\mathrm{CO})_{5}, \mathrm{Fe}_{2}(\mathrm{CO})_{9}$, $\mathrm{Fe}(\mathrm{CO})_{4} \mathrm{AA}, \mathrm{Mo}(\mathrm{CO})_{4}\left(\mathrm{CH}_{3} \mathrm{CN}\right)_{2}, \mathrm{Mn}_{2}(\mathrm{CO})_{10}$ und $\mathrm{C}_{5} \mathrm{H}_{5} \mathrm{Mn}(\mathrm{CO})_{3}$ als Inhibitoren wirken.

Bei Zugabe von $\mathrm{CCl}_{4}$ zu Mo(CO) ${ }_{6}$ bzw. Mo $(\mathrm{CO})_{5} \mathrm{Py}$ gelöst in $\mathrm{ÄA}$ tritt eine wesentliche Erhöhung der Polymerisations-Geschwindigkeit auf. Die Ergebnisse der thermischen und photochemischen Versuche können zwanglos mit dem von BAMford und Finch am Methylmethacrylat aufgestellten Reaktionsmechanismus interpretiert werden.
\end{abstract}

Wie seit langem bekannt ist, sind gewisse Metallcarbonyle und Metallcarbonyl-Derivate ausgezeichnete Katalysatoren für die verschiedensten organischen Reaktionen. Genauere Untersuchungen über den möglichen Reaktionsmechanismus ergaben den Hinweis, daß oft gerade die instabilsten Derivate oder instabile Reaktionsprodukte von Metallcarbonylen die größte Aktivität besitzen.

Metallcarbonyl-Derivate der allgemeinen Formel $\mathrm{YM}(\mathrm{CO})_{\mathrm{x}-1} \mathrm{D}$, wobei $\mathrm{Y}$ ein Ligand oder $\mathrm{CO}$ ist, sind nun photochemisch durch UV-Bestrahlung von Metallcarbonylen oder deren Derivate in Gegenwart von "n" ${ }^{1}$ oder ", $\tau^{\prime 2} 2$ Donatoren (D) leicht zugänglich ${ }^{3}$, selbst wenn D ein schwacher Elektronendonator ist. Auf diese Weise können, wenigstens in Lösung, relativ instabile Metallcarbonyl-Derivate erhalten werden, welche als Katalysatoren geeignet sind. In diesem Zusammenhang untersuchten wir auch die Möglichkeit Äthylacrylat $\mathrm{CH}_{2}=\mathrm{CH} \cdot \mathrm{COOC}_{2} \mathrm{H}_{5}$ (im folgenden $\ddot{\mathrm{A} A}$ abgekürzt) mit Metallcarbonylen oder deren Derivaten zu polymerisieren. Dabei

1 W. Strohmeier u. Kl. Gerlach, Z. Naturforschg. 15 b, 413 [1960]; Chem. Ber. 94, 398 [1961].

2 E. O. Fischer, H. P. Kögler u. P. Kuzel, Chem. Ber. 93. 3006 [1960]. interssierte gleichzeitig die Frage, ob bei einer eventuellen Polymerisations-Beschleunigung unter dem Einfluß des Metallcarbonyl-Derivates ein Unterschied besteht, falls dieses in Substanz eingesetzt wird oder erst in situ im Monomeren thermisch oder photochemisch erzeugt wird.

\section{A. Thermische und photochemische Polymerisation von $\ddot{A} A$}

Um den Einfluß von Zusätzen auf die Polymerisations-Geschwindigkeit des $\overparen{A A}$ zu erkennen, wurde zunächst die thermische und photochemische Polymerisation des reinen $\ddot{A} A$ untersucht, wobei als Maß für die Polymerisations-Geschwindigkeit die Ausbeute an Polymer in einer bestimmten Zeit genommen wurde. Die Charakterisierung des Produktes geschah durch Bestimmung des Schmelzpunktes und der Grenzviskositätszahl $[\eta]$, aus welcher sich das ungefähre Mol.-Gew. berechnen ließ. Die Ergebnisse sind in Tab. 1 zusammengestellt. Ausführliche Arbeiten über die thermische Polymerisation von $\ddot{\mathrm{A}}$ in

\footnotetext{
${ }^{3}$ Zusammenfassende Darstellung: W. Strohmeier, Angew. Chem., im Druck.

${ }^{4}$ H. Staudinger u. E. Trommsdorff, Liebigs Ann. Chem. 502, 201 [1933].
} 